\title{
URNA Y CANOA FUNERARIAS. UNA SEPULTURA DOBLE EXCAVADA EN PADRE LAS CASAS, PROVINCIA DE CAUTIN, IX REGION, CHILE
}

Américo Gordon

\section{INTRODUCCION}

En la zona centro-sur de Chile, entre los paralelos $37^{\circ}$ y $39^{\circ}$ Lat. S, se reconocieron sepulturas bajo elevados túmulos, en cistas construiclas de piedras, en urnas de greda cocida, en troncos de árboles ahuecados - "canoa" - y sin protección firme del cadáver. Los contextos inejor conocidos son de las sepultaciones en "canoa" descritos por Latcham (1928), Menghin (1962), Van de Maele (1968), Gordon, Madrid, Monleon (1972), además de algunas sepulturas en cistas referidas por Latcham (1928), Gusinde (1948), Reymon (1971). Casi un centenar de urnas han sido descritas por Bullock (1955 y 1970).

Documentos que datan de la época de la conquista española del país atestiguan el empleo de ataúdes labrados de troncos por la población autóctona, tradición funeraria que se conservó hasta las primeras décadas del presente siglo. La mayoría de las urnas no procede de excavaciones arqueológicas controladas y de las reliquias asociadas se conocen solamente algunas vasijas y adornos personales. Sin suficientes datos confiables y sin fechados radiocarbónicos, Bullock (Op. cit.), adscribió esta tradición funeraria a una cultura premapuche. Menghin (OP. cit.), estudió la alfarería que se halló junto a las urnas y la asignó a dos fases culturales. La primera, Vergelense $I$, sería anterior a la invasión incaica; y la segunda, Vergelense II, posterior a la conquista hispana.
E1 hallazgo de una sepultura doble, que contenía una urna dentro de una "canoa" funeraria y la fecha radiocarbónica, aducen la primera evidencia que ambas tradiciones funerarias se practicaron simultáneamente por la misma comunidad indigena en épocas pre. hispánicas.

\section{CAPITULO I}

\section{HISTORIA DEL HALLAZGO}

El descubrimiento de una sepultura doble en Padre Las Casas se clebió al azar. Mientras Aureliano Villanueva* y su hijo cavaban un hoyo para la colocación de un poste en la sede de la Sociedad Cultural y Socorros Mutuos*, su pala topó con el borde de una gran vasiji. Afortunadamente la dejaron en su lugar y avisaron del hallazgo a nuestro Grupo de Investigaciones Indígenas de la Frontera, que opera bajo el patrocinio de la Universidad de Chile, Sede Tenuco. La excavación y el estudio se realizó en colaboración con el Museo Nacional de Historia Natural de San. tiago.

Después de verificar la presencia de una

- Agradecemos especialmente a los señores Aureliano y Juan Villanueva por su abnegada colaboración como también al Directorio y socios de la Sociedad Cultural y Socorros Mutuos por las amplias facilidades otorgadas para ejecutar la excavación. 
una tuneraria decidimos realizar una exaración de salvamento, en la espresana que la minuciosa obserrarión y cl esturlin del contexto finerario ofrecieran. eventualnente, una oportunidad de ampjiar los ciscasos datos arqueológicos acerca de esta traclición mortuoria. Nuestro esfucrzo se ha visto ampliamente recompenardo y las esperanzas sobrepasadas con el hajlizgo de unil segunda sepultura en "canoa" y la comprobación de que la urna si hallaba estrechamente asocia. da it 1.! "camosi".

El sitio del hadiazgo se enchentra en pleno sector residencial edíicado, lo que impidio la ampliación del área de excavación.

\section{CAPITLLO H}

\section{UBICICION GEOGRAFICA}

Padre Las Casas (30 $16^{\prime}$ Lat. S, i2 $35^{\prime}$ Long. W, $111 \mathrm{~m}$ s.n.m.), corresponde al sector sureste de la ciudi..d de Temuco, proviticia de Cautín, IX Región. El lugar del hallazgo está ubicado a $25 \mathrm{~m}$ de altura y aproximadancn. te a 800 in de distancia de la orilla sur clel lío Cautin. Según nuestros informantes, halbitontes mapuches del barrio, el paraje se denominaba originalmente Llamaullin (Lugar de Camarones).

\section{GEOMORFOLOGIA}

De acuerdo con lal descripción de corfo (1964:259), los suclos son de posición intermedia, plano deposicional, ondulado a quebrado. Material de origen: cenizas volcánicas. asociadas a conglomerados. Drenaje cxterno e interno: medio. Erosión: moderadi.

\section{CIIMA}

EI climal presenta precipitaciones invernales, durante seis meses del año (abril a septienlbrc) éstas sobrepasan el promedio mensual de $110 \mathrm{~m} / \mathrm{m}$. Los meses de verano (diciem. bre a febrero) pueden ser notablemente se- cos. En el año 1972 la temperatura mínima se registró en al mes de juïio con $-5,4^{\circ} \mathrm{C}$ y lis máxima, en enero. con $33,2^{\circ} \mathrm{C}$.

\section{FLORA Y FAUNA}

De la selva austral, que cubría la región a principios de nuestro siglo, solamente se conservan unas islas testigos en los valles de la cordillera y ell los cerros Sílol. La flora autóciona consiste principalinnte de especies de álboles siempre vercles de lats lamilias de conileris, minticeas y haticents. Aibuncla, además, una regetación monor de arbustos, mientras que lianas de las plantas repadolas hacen impenerable al borgue nativo. Matorrales y hierbas altas cubien los temenos pantanosos.

De acuerdo con la estación del año varía conidcrablemente la fauna. que inchye una gran ralictad om nitológica. En lon rios Cautín, Tultén y sus afluentes abumalan los peces. mientras en los lugiales pantanosos los camarones.

El sistemla ecológico pre y protohistórico no sulitó alteraciones sensible, por la economía de subsistencia de la población nativa. La tala y el roce del boeque se mantuvo equilibraclo con el crccimiento naturit. La rápidá fommación de renovales de bosques, en terrenos de cultivos abanclonados, evitó la erosión. el clesplazamiento : la extinción de especies faunísticas ! botánicas, tal como aconteció en el curso del siglo $\mathrm{xx}$.

C.PPITUIO III

\section{ANTECEDENTES HISTORICOS $Y$ AROUEOLOGICOS}

\section{a) Referencias históricas a sepulturas en "artesas" $v$ "artesones"}

Disponemos de documentos que datan de los comienzos de la época de la Conquista, que atestiguan el empleo de ataúcles de madera por la población indígena. El primer cronis. 
ta de Chile, Gerónimo de Bibar, en su Cró. nica y Relacion Copiosa y Verdadera de los Reynos de Chile (1558), en el Capítulo $\mathrm{CV}$ "... que trata de las costumbres y ceremonias de la gente de la ciudad de Concepción", escribe:

"Sus enterramientos es en el campo con las ceremonias de los de Mapocho. Otros se entierran a las puertas de sus casas en un alto, que es hecho de dos horcones gruesos, y ponen clos a manera de artesas angostas arriba. Métenle en uno y cúbrenlo con la otra. Este es su entierro y sepultación de algunos principales". (BIBAR, 1966: 156).

A principios del siglo xvir, González de Nájera menciona la misma práctica de disponer de los difuntos:

"Los enterramientos de los caciques son algo levantados de la tierra, porque ponen sus cuerpos entre dos grandes artesones cerrados, hueco con hueco, y encajado entre dos árboles, juntos, sobre fuertes horcones, y éste es el fin de sus vidas y paradero de sus cuerpos". (Gonzál.ez, 1971: 49).

Resulta notable de observar que ambos cronistas se relieren en términos casi iclénticos al empleo de "artesas" y de "artesones" para la sepultación de algunos dignatarios indígenas.

\section{b) "Canoa" funeraria.}

\section{Investigaciones arqueológicas}

Evidentemente, corresponde a la "artesa" o "artesón" de los cronistas la "canoa" funera. ria de los mapuches. Tal tipo de ataúd se confeccionó de un tronco de árbol de roble o de coigüe, partido a lo largo y las dos partes vaciadas formaban el fondo y la tapa de la "canoa". El bote mapuche es de forma similar, pero se distingue del primero por carecer de tapa. El idioma mapuche distingue perfectamente a los dos artefactos, de acuer- do con su lunción. Denomina wampu o wampo al bote y trolof al ataúcl.

Latcham (1915), asigna el uso de la "ca noa" funeraria al pueblo mapuche, que, según su teoria, a la llegada de los conquistadoces hispanos ocupaba el territorio entre los rio; Itatá y el Toltén. Sostiene que era el único pueblo en Chile.

"quienes ciesde la época de la conquista y intes enterraban sus muertos en ataúdes de madera en forma de canoas llamadas trolof. (LATChAM, 1936: 86).

Supone, además, que la costumbre de sepultar en "ranoas" se extendió a la región situada al sur del rio Tolı́n, solamente en la época colonial y que la inhumación del ataúd, en rez de su colocación sobre horcones, seria la imitación de la costumbre funeraria cristiana.

Las prácticas funerarias mapuches, a principion de nuestro siglo, describe Tomás Guevara en los siguientes términos:

"El cadáver quedaba expuesto al fuego en un aparato de varas delgadas que iban amarradas paralelamente, cuando sobrevino la descomposición cadavérica, colocábanlo dentro de dos canoas, sobrepuesta una en olra (wampu) (Gufvara, 1916: 130).

Según nuestros informantes, el trolof estaba en uso hasta en las primeras décadas del sigho. En la actualidad, el atáud se com. pra confeccionado de tablats aserradas.

\section{c) Sepulturas en "canoas". \\ Antecedentes arqueológicos}

A pesar de la amplia difusión regional y profundidad cronológica de la sepultación en "canoas" funerarias, solamente unas pocas han sido identificadas en las excavaciones arqueológicas. El fenómeno se debe, principalmente, según suponemos, a la dificultad de advertir en el suelo húmedo los vestigios de 
la madera descompuesta. Las sepulturas cn rollcos ahuecados se reconocieron en las excavaciones arqueologicas solanicinte ell los casos en que se llatló un trozo de madera bien conservada en la tumba, que lia podido ser idenuticado como resto del ataúd. Fue por primera vez en nuestras excavaciones ell el cementerio indigena de Gorbea (Gordon y otros, 1971: jus;, que reconocimos seplilturas en "canoas" sll encontrar ningúl trozo de madera basándose solamente en los indicios que revelan la presencia de madtra descompuesta: el color y la textura del suelo.

En la localidad de Huitag, al norte del lago Calafquén, Menghin (1962), excavó algunas sepulturas y en una identificó la tapa àe la "canoa" en buen estado de conservación. Encontró al lado de un cráneo un tupu de cobre con aguja de hierro, un puco fragmentado y un vaso negro de boca anclia. En el mismo cementerio excavó un jarrito con incrustaciones de loza eúropea. Adscribió los hallazgos a la primera mitad del siglo xvill.

Entre las localidades de San José y Madre de Dios, en Puile, Van de Maele (1968), excavó inhumaciones con $\mathrm{y}$ sin protección firme, algunas superpuestas. Entre el ajuar funerario encontró objetos alfareros, collares de piedras, de conchas y de vidrio, abundaban los objetos de origen europeo, los platos de loza, ollas y cuchillos de hierro y algunas monedas de plata. Según el investigador, las inhumaciones se habrian efectuado liasta el año de 1870 .

Nosotros presentamos un informe al vi Congreso de Arqueología Chilena (Gordon, Madrid, Monleon, 1971) sobre las excavaciones realizadas en Gorbea (sitio Go-3). En este cementerio las "canoas" funerarias se hallan a veces superpuestas, parcialmente conservadas o completamente descompuestas. Algunas guardan todavía restos óseos.

El ajuar funerario consistió en ollas, jarros, cántaros y grandes tinajas. Los jarros y cántaros están decorados en gran porcentaje con partículas de loza incrustadas en la boca y el cuerpo de las vasijas. En unos pocos ejellplares está presente el tipo Valdivia rojo sobre blanco. Entre la alfarería modelada se desta. ca un jarro antropomorlo y otros zooniol tos. Los adornos personales consistían en aros cuadrangulares, coronas circulares, tupus de cobre y de plata con clavo de hierro, una cru de metal puede haber formado parte de un rosario, abundan las cuentas de vidrio de variadas formas y colores.

Se observa frecuentemente la fractura intencional y la pertoración mágico-ritual de ceramios para inutilizarlos.

Cironológicamente atribuimos la mayor frecuencia de las sepultaciones hacia fines del siglo $\mathrm{x} 1 \mathrm{x}$, en una lase tardía de la cultura mapuche.

En el cementerio de Membrillo, Reynond (1971) encontró, dentro de una cista formala de piedras de laja, los restos de una pequeña "canoa". Huesos humanos, clavos cle hierro, cuentas de vidrio y adornos de metal șe hallaron en el lugar. El cementerio se ubicó entre fines del siglo xviII y principios del xix.

Por falta de mayores antecedentes y de fechas absolutas los investigadores atribuyen, generalmente, los cementerios con restos de madera o con huesos humanos asociados a objetos de procedencia europea, a los siglos xvili y xix.

\section{d) Sepulturas en urnas funerarias}

Carecemos de toda referencia bibliográfica $u$ oral a la tradición indígena de emplear urnas de greda cocida con fínes funerarios en la región centro-sur del pais. Entre la población autóctona, aparentemente, no existe recuerdo de su empleo y se desconoce la palabra con lia cual lic denominaban.

Sin embargo, en el año 1912, en relación con el problema que representaba para los investigadores la colocación del cadáver en la urna, Guevara escribe a Latcham:

"He recogido varias tradiciones que me informan que los cadáveres se destrozaban 
para meterlos adentro. No se han hallado críneos, al menos que yo sepa.

Me lundo en que cocían los indios estos rasos después de poner dentro el cadáver destrozado porque algunos tienen la boca muy estrecha" (LiтchaM, 1928: 41).

De ser verídica la afirmación de Guevara que recogió información referente a la colocacoón del cadáver en la urnit, entonces se podría suponer que a principios del siglo existian personas que tenían conocimiento de la sepultación en umas. Parcce muy poco probable que "los indios cocían estos vasos después de colocar el cadáver adentro". Aparentemente Guevara confunde las tinajas de boca estre. (ha, que servian para guardar bebidas y eventualmente granos, con las urnas funerarias que tienen la boca ancha.

\section{e) Referencias arqueo!ógicas a urnas funerarias}

E1 historiador e investigador de nuestras culturas indígenas, J. T. Medina (1882) excavó en La Patagüilla (Prov. de Curicó) una vasija que contenía el cráneo de un feto, semillas y restos de roedores en inal estado de conservación. Menciona otro hallazgo similar procedente de la lracienda de la Compañía (Graneros). En este caso, una olla contenía los restos de un niño y algunas chaquiras. Medina (1952: 270) supone que la práctica de este tipo de sepultación se debía a la adaptación de costumbres peruanas.

En una excavación arqueológica realizada a corta distancia de la playa de Llolleo, Oyarzún halló seis esqucletos dentro de urnas.

"Los cadáveres habían sido sepultados de una manera nueva, fuera de uso entre los aborígenes y los actuales indios araucanos. En unos seis esqueletos exhumados vi que todos ellos estaban dentro de ollas de greda de unos $60 \mathrm{~cm}$. de alto. Estas ollas estaban todas ellas quebradas... los cadáveres estaban en cuclillas, las rodillas alcan- zaban ai mentón y los miembros supurcres tenian las manos a nive! de los hombros. Dentro de las mismas ollas o urnas se encontraban acompañando al cadáver uno, dos y hasta tres cantaritos de greda cocida y sin dibujos" (OYarzín, 1910: 14).

Limentablemente, en la época de esta excavacion no era costumbre recolectar los fraginentos cle greda y por tal motivo desconocemos el tipo de urnas empleadas en la costa de la zona central.

La primera evidencia de la existencia de urnas funerarias en la región contro sur representó una vasija hallada en Angol, con los restos de un niño en su interior. Guevara al referirse al hallazgo sostiene que la urna:

“... es de origen antiguo porque en los cementerios de data posterior no se ha des. enterrado ninguna" (Guevara, 1908: 262).

$Y$, en relación con la relativa posición cronológica de las urnas, Guevara en carta dirigida a Latcham expresa:

"Opinamos que estos vasos funerarios fueron anteriores a los wampus de madera". (Latcham, 1928: 41).

Des:lortunadamente, el investigador omite precisar cuáles son, según su criterio, los cementerios "de data posterior" y el material arqueológico asociado, que le permiten postular la antigüeclad de las inhumaciones en urnas y su empleo anterior al de la "canoa" flineraria.

Dillman S. Bullock recolectó en el museo que actualmente lleva su nombre, en la ciudad de Angol, cerca de un centenar de urnas halladas en la región centro-sur. Sus publicaciones (1955 y 1970) son la fuente más completa sobre la materia. Casi la totalidad de las urnas fueron encontradas por campesinos mientras ejecutaban trabajos agrícolas y sola. mente unos pocos han sido excavados por Bullock y sus contextos reg:strados. Bullock ob- 
servó también que los sitios donde fueron hechos los hallazgos siempre presentaban gran cantidad de fragmentos alfareros y objetos líticos, lo que le permitía presunir que las ur. nas se enterraban en terrenos habitacionales.

El director del museo de Angol aceptó la teoría de Latcham acerca de la invasión de los mapuches a la región entre los rfos Itata y Toltén. Adscribió el empleo de las urnas al pueblo que ocupaba dicho territorio antes de la llegada de los mapuches (Blllock, 1955). Pero, debido a nuevas evidencias, posteriormente expresó:

"Creemos que el uso general de las sepulturas en urnas era costumbre de los kofkeche y no de los mapuches.

Sin embargo, es muy posible que muchos indígenas siguieron la costumbre antigua... de usar urnas de loza, en especial para niños y hasta en los tiempos moder. nos" (Bullock, 1970: 64).

La teoría de Bullock acerca de las urnas ha sido analizadla por Menghin (1962), quien llamó la atención sobre las diferencias existentes en la alfarería asociada a este tipo de inhumaciones y observó que:

“... se trata evidentemente de dos componentes heterogéneos",

y supone que el grupo más antiguo incluye:

"jarros asimétricos, muchas veces con cuello cigüeñal y con decoraciones bastante peculiares",

mientras el grupo más reciente se caracteriza por:

“jarros simétricos, cuya forma y decoración ya los acerca a la famosa cerámica postcolombina de Valdivia" (Menghin, 1962: 17).

A base de la tipología alfarera, Menghin subdivide al complejo cultural, que presenta sepulturas en urnas, en dos etapas:
1400 al 1450 D.C. Vergelense I inmediatamen. te preinca.

1450 al 1550 D.C. Vergelense in coincide con la dominación incaica en Chile y el comienzo de la conquista española.

En relación con la presente cronologia tentativa cabe recordar que las secuencias se fundamentan en la diferencia tipológica alfarera, materia, a su vez, poco conocida en sus fases de desarrollo.

\section{CAPitulo iv}

\section{EXCAVACION Y TECNICA}

\section{a) Trabajos previos}

A nuestra llegada al lugar del hallazgo observamos un hoyo recién abierto, que, casualmente, coincidió con la boca de una urna funeraria. A través de la abertura su interior completamente vaciado era visible y se adver. tía que estaba fuertemente fracturado. Antes de emprender su excavación revisamos la tierra extralda de su interior por sus descubridores y recogimos cuatro dientes humanos. Con el propósito de evitar el desprendimiento de los fragmentos y la desintegración de la urna, la rellenamos con tierra.

Para la excavación demarcamos dos cuadriculas: una menor de $0,80 \times 0,80 \mathrm{~m}$., la que encerraba la urna en su medio y otra homóloga, de $2 \times 2 \mathrm{~m}$. Mediante este ordenamiento excavamos por capas artificiales de $0,10 \mathrm{~m}$. cuatro trincheras alrededor de la urna, que quedó sin excavar y protegida por una columna de tierra, durante la primera fase de los trabajos.

\section{b) Estratigrafia}

En el curso de la excavación distinguimos tres estratos de tierra. Los dos primeros están formados por trumao, mientras el tercero corres- 
ponde a un conglomerado de cascajo. El Estrato $\mathrm{I}$, entre 0 a $22 \mathrm{~cm}$. de profundidad, es de color negro-pardusco con gran porcentaje de material orgánico descompuesto. El Estra. to II, entre 22 y $86 \mathrm{~cm}$., es de color pardo-rojizo en la parte superior y pardo amarillento hacia la base del estrato. El Estrato III aparece a $86 \mathrm{~cm}$. de profundidad y corresponde a un conglomerado de cascajo de consistencia dura, compacta, de color gris-blanquecino.

Los dos primeros estratos contienen hasta $60 \mathrm{~cm}$. de profundidad material cultural. El tercer estrato se presentó estéril desde el punto de vista arqueológico, salvo una cavidad de poca profundidad, labrada para la colocación del ataúd de tipo "canoa".

\section{C.APITULO $\mathrm{v}$}

\section{MATERIAL ARQUEOLOGICO}

La excavación estratigráfica rindió abundante y variado material cultural. El primer estrato contenía material orgánico: madera, carbón vegetal, huesos de animales, dientes de caba1lo, cuescos de durazno; el material inorgánico es también de origen moderno: trozos de metal, clavos, alambre de cobre, monedas de los años 1960 a 1966, vidrio, material sintético y trozos de tejas. Suponemos que los pocos objetos de origen indígena son intrusivos en el estrato, removidos de mayores profundidades al realizar construcciones y efectuar la colocación de cañerías en el sitio.

Los hallazgos del segundo estrato pertenecen a industrias líticas y alfareras indígenas. Observamos también algunos bolsones de relleno, éstos correspondían a emplazamientos de postes redondos y cuadrangulares. Sin excepción, rindieron moderno material cultural, similar al primer estrato.

Incluidos en el segundo y tercer estratos se hallaron lis sepulturas que son la materia del presente informe.

\section{LAS SEPULTURAS}

A1 alcanzar la excavación la profundidad de
$056 \mathrm{~m}$. se observó en la trínchera este uita ancha franja de tierra negra, de contexto blando, que se destacó notablemente del suelo de trumao circundante, de color claro y contexto más duro. Con el propósito de delimitar dicha franja en toda su extensión se procedió a extender las trincheras sur y oeste. Después de efectuar la ampliación y excavando en la franja negra a $0,72 \mathrm{~m}$. de profundidad, en la trinchera oeste se ubicó la boca de una olla. En sus inmediaciones, pero a un nivel inferior yacía un cráneo humano. Estos hallazgos evi. denciaban que la oscura franja de tierra, que se extendía a ambos lados de la urna, correspondía a una segunda sepultura.

En la tierra negra se destacaron dos vetas paralelas de color pardo-violáceo, aproximadamente de $2,5 \mathrm{~cm}$. de ancho y distantes en $48 \mathrm{~cm}$. entre sí. Según nuestra experiencia en excavaciones en la región, indicaban la presencia de madera descompuesta y en este caso, correspondían al borde superior del ataúd cuya forma y dimensiones fueron identificados en el curso de los trabajos subsiguientes.

Alredecior de la urna se observó un cambio en la composición del suelo, su color era pardusco y su contexto compacto. Tal diferencia. dentro de la sepultura, evidenciaba la práct: ca de una fosa secundaria, cavada para la colocación de la urna, en medio de la primera sepultura y rellenada posteriormente.

Después de excavar las cuatro trincheras hasta la capa de cascajo, se procedió con la remoción de la columna que encerraba la urna.

Por existir dos sepultaciones de diferentes tradiciones funerarias, describiremos cada cual por separado. Denominamos Sepultura No 1, la inhumación practicada en el ataúd de madera, en un tronco de árbol ahuecado, denominado comúnmente "canoa", y Sepultura No 2, la efectuada en una urna de greda cocida.

\section{Sepultura No 1}

La excavación por delgadas capas y la constante observación de la tierra negra permitió 


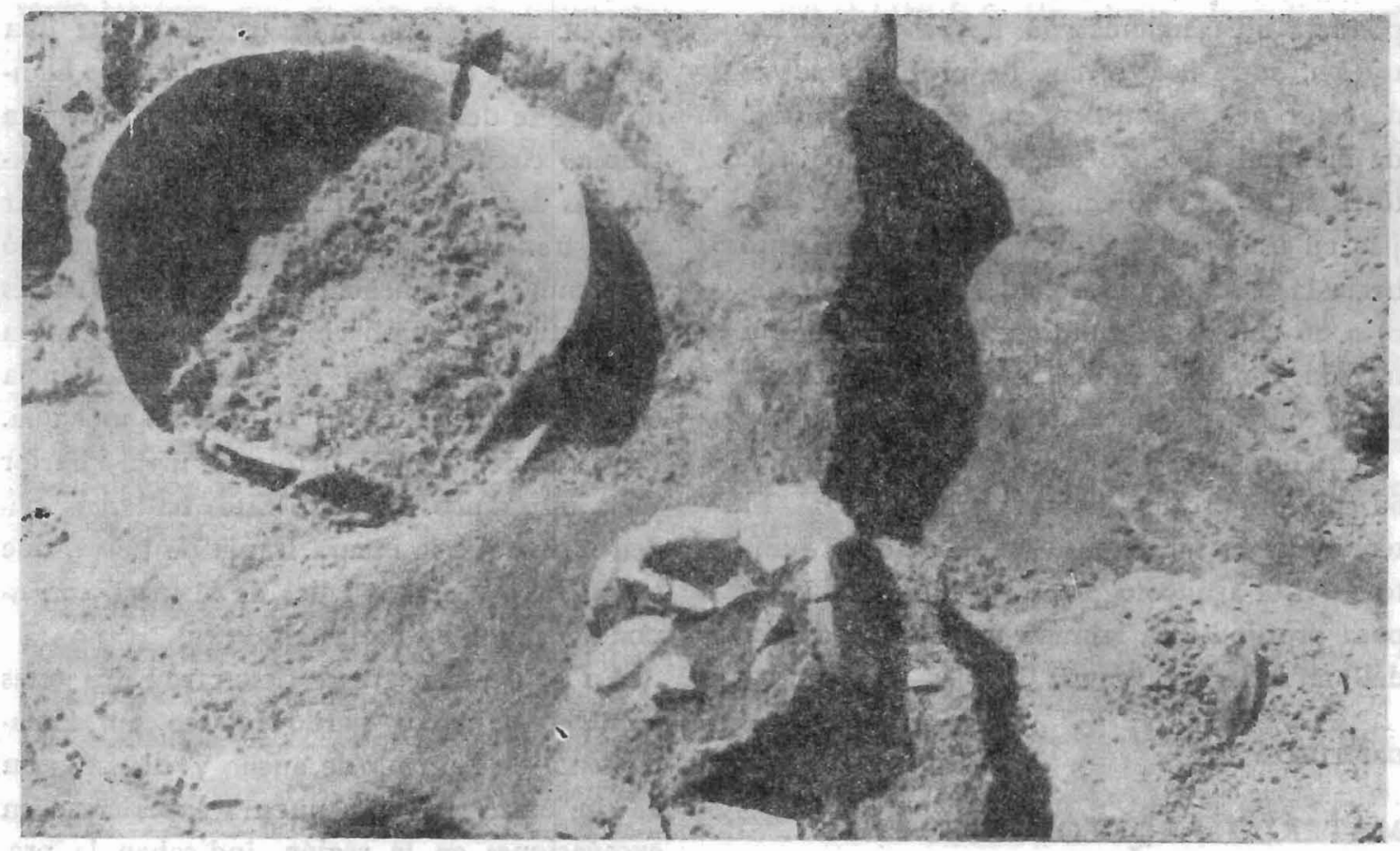

Foto No 1. Boca de la urna. Cántaro fracturado intencionalmente. Relleno de un poste cuadrangular; quijada de oveja. (Foto: W. Enrique Eilers)

reconocer los vestigios que deja la madera descompuesta en el suelo y que difiere en su composición, densidad y color de la tierra circundante. La observación de estas características condujo al reconocimiento de la forma y dimensiones del ataúd, del trolof mapuche, referido en los estudios etnológicos y arqueológicos como "canoa" funeraria. Para su colocación se labró una fosa a través de la capa de trumao y una hendidura de $0,12 \mathrm{~m}$. y de $2 \mathrm{~m}$. de largo en el duro cascajo.

En la sepultura se encontró un cráneo en mal estado de conservación. A $52 \mathrm{~cm}$. de distância estaba colacada, dentro de la fosa, la urna. Adosados al lado este de la urna, a $56^{\circ}$ $\mathrm{cm}$. de profundidad, ubicamos en posición vertical las siguientes osamentas humanas*: diáfísis tibia derecha junto al diáfisis fémur izquierdo, diáfisis fémur derecho junto a otro

- Agradecemos a la señorita Consuelo Valdés Ch. y al profesor Juan Munizaga, del Departamento de Antropología de la Universidad de Chile, Santiago, su colaboración de efectuar el estudio de los restos óseos. hueso largo no identificado, posiblemente la tibia izquierda. El cambio del lado derecho al lado izquierdo y del lado izquierdo al dere. cho de los fémures y su posición vertical indica que éstos han sido alejados de su posición anatómica.

A ambos lados del cráneo se encontraron adornos personales, aros cuadrangulares enmarcados en circulares. Pequeñas, delgadísimas cuentas de piedras negras y blancas, llancas, y un pequeño trozo de cobre yacían des. parramados delante del cráneo. Debajo del cráneo, en el fondo de la sepultura, recolectamos trocitos de madera y carbón vegetal.

Para verificar que las dos vetas observadas efectivamente correspondian a madera en descomposición, recolectamos muestras de tierra en tres diferentes partes de la tumba. La Muestra No procedía de la veta de color pardo-violáceo. Esta, según nuestra suposición, correspondía a la "canoa" descompuestá. La Muestra No 2 fue recolectada en el interior de la sepultura, mientras la Muestra No 3 se sacó del suelo, fuera de la sepultura. Hipo- 
téticamente, el análisis de la Muestra $\mathrm{N}^{\circ} \mathrm{l}$ debia inclicar el mayor conteniclo de materia orgánica. El cstudio realizado en la Universidad Austrai de Valdivia dio el siguiente resultado*:

\begin{tabular}{|c|c|c|c|c|}
\hline & & & UESTI & \\
\hline & & NOI & No 2 & $\operatorname{Nos} 3$ \\
\hline $\mathrm{pH}$ & 1: 2.5 agua & 6.20 & 6.45 & 6.80 \\
\hline$r_{n} \mathrm{C}$. & orgánico (Walkley Black) & 3.28 & 2.96 & 2.42 \\
\hline$m_{0} M$ & $0 .(0, \times 1.721)$ & 5.70 & 5.10 & 4.20 \\
\hline
\end{tabular}

El análisis de las muestras comprobó que la Muestra 1 ? 1, extraida cle la veta de color, dejada por la madera del ataúd, efectiramente contenía $36 \%$ más materia orgánica que el suelo fuera de la sepultura y demostró tener el menor índice de acidez.

\section{Sepultura $\mathrm{N} \div 2$}

Los descubridores de la urna encontraron en su interior ocho dientes humanos, el arco de suspensión de un aro de cobre, un cantarito con engobe rojo, además de algunos fragmenlos alfarcros y líticos. La urna se halló sin ta$\mathrm{pa}$, u borde estaba a $042 \mathrm{~m}$ - desde la superfic.e del terreno y descansaba sobre siete piedras rodadas colocadas alrededor de su fondo para mantencrla en posición vertical.

CADITLLO VI

\section{LOS HALLAZGOS}

a) Fragmentos alfareros $y$ objetos liticos

A continuación indicamos las cantidades de fragm $€ n t o s$ alfareros y objetos líticos rescatados; su estudio se halla en las páginas de esta publicación: 41 a 49 y 51 a 59 .

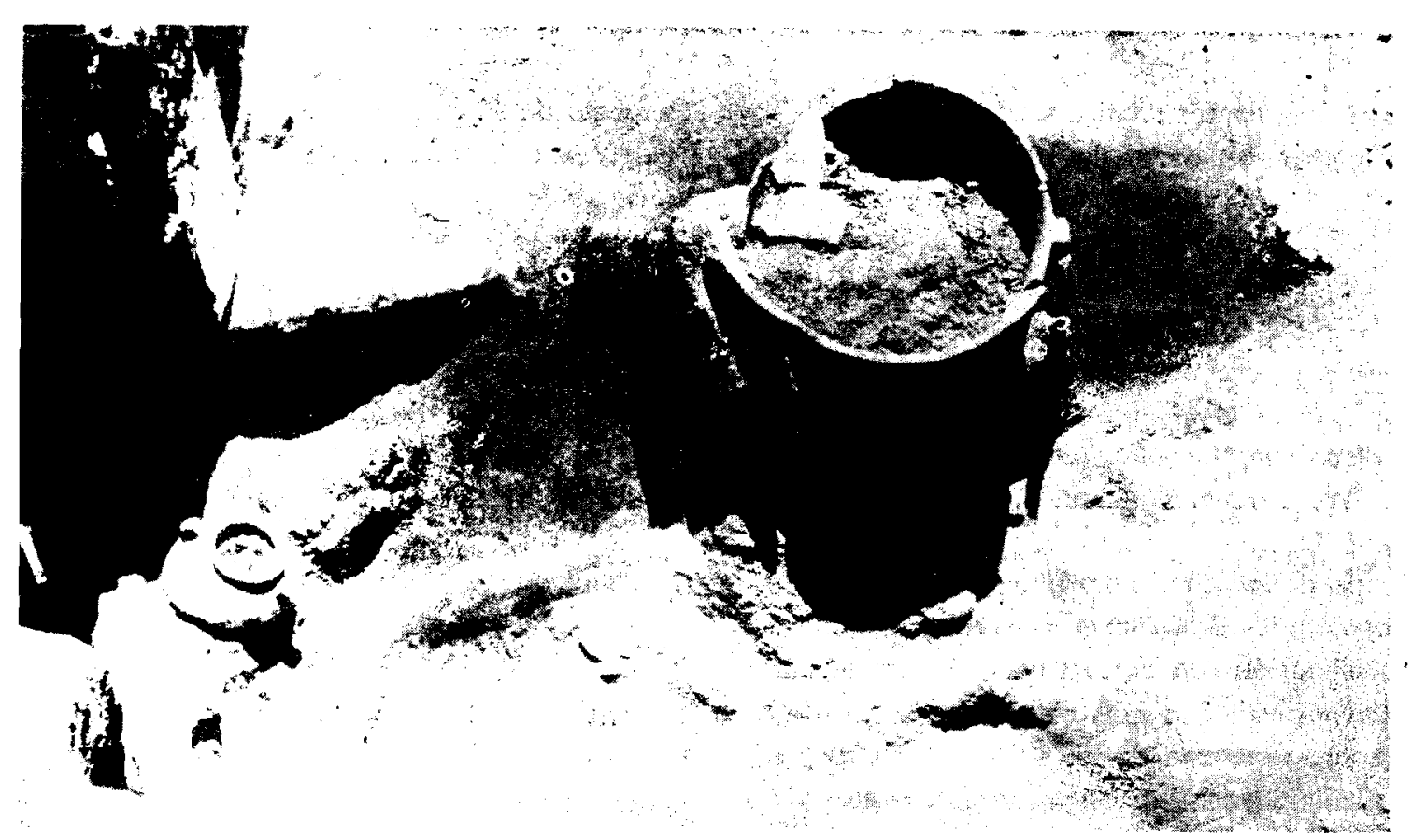

Foto $N^{2}$ 2. Sepultura doble. Obscruense Jas diáfisis fémur izquicrdo junto a la tibia derecha.

(Lado der. de la urna). (Foto, W. Enrigue Eilers) **

*Agradecemos al señor Ing. Agr. Pablo Alister, del sAG de Temuco su gentil colaboración, y al scñor Ing. Agr., Gastón Arcaus, Jefe del Laboratorio de Edafología, el estudio de las muestras del suelo.
**Agradecemos al Sr. W. Enrique Eilcrs, profesor de Fotografia y Cinematografía, por la realización de las secucncias fotográficas. 


\begin{tabular}{cccccc}
\hline \multirow{2}{*}{ Estrato } & $\begin{array}{c}\text { Profundidad } \\
\mathrm{cm}\end{array}$ & $\begin{array}{c}\text { Fragmextos } \\
\text { alfareros }\end{array}$ & $\%$ & $\begin{array}{c}\text { Objetos } \\
\text { liticos }\end{array}$ & $\%$ \\
\hline I & $0-10$ & 3 & 0.72 & 5 & 3.17 \\
- & $10-20$ & 35 & 8.37 & 16 & 10.12 \\
II & $20-30$ & 129 & 30.85 & 58 & 36.72 \\
& $30-40$ & 141 & 33.72 & 54 & 34.17 \\
& $40-50$ & 78 & 18.68 & 18 & 11.39 \\
& $50-60$ & 32 & 7.66 & 7 & 4.43 \\
\cline { 3 - 6 } & Totales & 418 & 100.00 & 158 & 100.00 \\
\hline
\end{tabular}

b) O:la globular (Lám. 3. Fig. 2)

La olla, de $18 \mathrm{~cm}$. de altura, tiene el fondo convexo, corto cuello cilíndrico y posee dos asas verticales con protuberancias horizontales. La superficie externa ha sido alisada, pero se observan rayas dejadas por el artefacto alisador. Su color exterior es pardo oscuro y se encuentra parcialmente tiznado por el hollín.

\section{c) Jarro asimétrico (Lám. 3. Fig. 3)}

De los fragmentos recolectados ha sido posible reconstruir un jarro de $18 \mathrm{~cm}$. de alto, con cuerpo de forma ovalada horizontal, con base plana y cuello cilíndrico. Sobre el asa vertical existe una protuberancia horizontal. La superficie, poco alisada, de color gris-negruzco, se presenta muy erosionada.

d) Aros cuadrangulares (Lám. 3. Fig. 6) Aros circulares (Lám. 3. 'Fig. 5)

El tipo de aro cuadrangular, confeccionado de una plaquita de cobre, posee un amplio arco de suspensión con muesca en su base y termina en espiral. Los aros circulares de alambre de cobre muestran en sus extremos, de manera similar, espirales contrapuestos. Debido a la oxidación del metal se denota una ligera corrosión.

e) Llancas o cuentas de piedra

(Lám. 3. Fig. 7)

Las pequeñas, delgadísimas cuentas de piedra de menos de $3 \mathrm{~mm}$. de diámetro y de 0,26 a $0,37 \mathrm{~mm}$. de espesor, tienen superficies lisas, los cantos redondeados y el agujero bien centrado.

\section{f) Restos humanos}

El cráneo y los huesos largos han sido identificados como pertenecientes a un individuo adulto joven. (Ver Anexo 1)

\section{g) Urna funeraria (Lám. 3. Fig. 1)}

La urna, de $64 \mathrm{~cm}$. de alto y $44 \mathrm{~cm}$. de boca, es de cuerpo cilindrico ligeramente cóncavo en su tercio superior y ovoide en su parte inferior. La superficic alisada ha sido tratada con un baño de color gris-pardusco. Sobre las asas verticales existen protulerancias horizontales. A lo largo de una trizadura se practicaron cuatro perforaciones para repararla. Tipológicamente, pertenece al grupo más frecuente al sur del río Biobío.

\section{b) Cántaro tipo "pato"}

(Lám. 3. Fig. 4)

El cantarito, de solamente $7 \mathrm{~cm}$. de alto, es de forma compuesta, asimétrica, angular, fondo plano, con un asa vertical. La posición oblicua del cuello cilíndrico le da la apariencia de un "patito". Su superficie con engobe rojo se encuentra fuertemente erosionado.

\section{i) Restos humanos}

Los dientes encontrados en el interior de Ja 


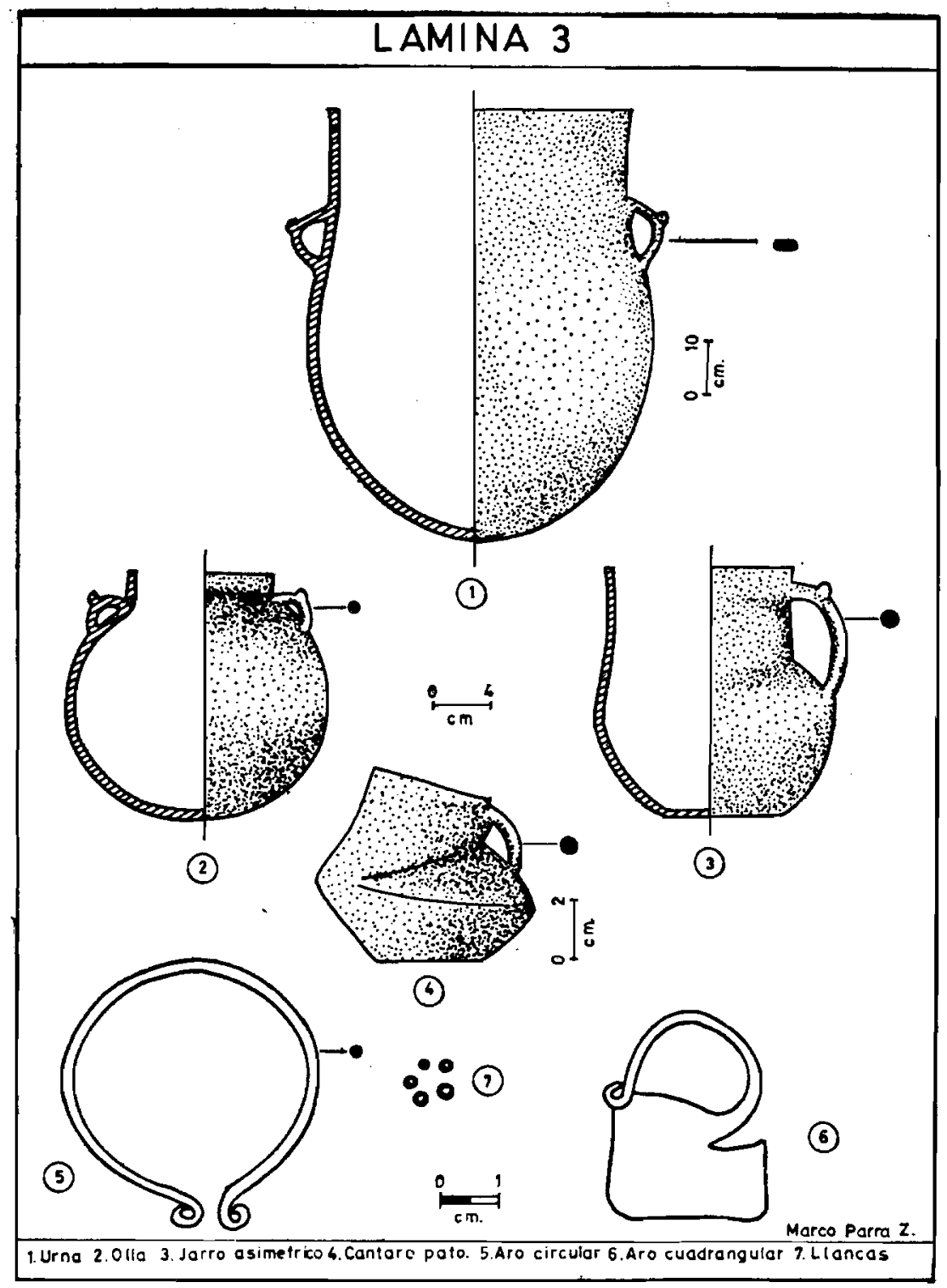

urna corresponden a un niño de aproximadamente cinco años de edad. (Ver Anexo 2)

\section{CAPITULO VII}

\section{DISCUSION Y CORRELACIONES}

El estudio del material excavado y del ritual observado, inducen al intento de correlacionar el contexto de las dos sepulturas con hallazgos similares y con tradiciones que persisten hasta el presente.
En la sepultura doble no se halló ninguna rasija decorada, aunque en casi todos los estratos existen fragmentos alfareros con engobe blanco con dibujos geométricos en color rojo o con engobe rojo con dibujos en blan. co. Los objetos alfareros del ajuar funerario corresponden a formas no registradas en la región.

La olla utilitaria se asemeja a la ollita miniatura encontrada en una de las urnas en Llolleo (Oyarzún, 1910: Fig. 11). El tipo es frecuente en Atacama (Latcham, 1928; 85; 9). 
Un jarro ovalido horizontal, pero con base convexa y superficie decorada se excavó en una tumba en Chiguayante; lia sido adscrito a una temprana época colonial (CHizelle y otros, 1966). Aunque el cantarito miniatura no tiene la clásica forma de "pito", informantes mapuches seña:aron que su forma sugiere a un patito. El profesor Martín Alonqueo Piutrín nos contó que el cántaro "pato" era un símbolo femenino, y como tal se depositaba en las tumbas de las mujeres. En el cementerio indígcna excavado en Gorbea, todos los cántaros clasificados dentro del tipo "pato" se hallaron en sepulturas de individuos de sexo femenino (Gordon, Ms.).

Al carácter ceremonial-religioso del cántaro "pato" o quetru se refieren Latcham (1924: 57) y Casamiquela (1972: 496). Uno de nuestros informantes señaló que a la celebración del nguillatun solamente las mujeres aportan el muday en jarro "pato" y que los varones no poseen tal tipo de vasija, aunque pueden emplearla.

Llama también la atención el tamaño reducido del cantarito. Oyarzún encontró vasijas miniaturas en las urnas de Llolleo. Bullock piensa que estos pequeños ceramios son juguetes, depositados junto con los niños. Sin embargo, Latcham los encontró en un conchal en Algarrobo, en sepulturas de adultos, al igual que en Cobija, y estima que las miniaturas alfareras corresponden a:

“piezas funerarias colocadas en tumbas en vez de las piezas grandes de uso diario y en representación de ellas" (LATchaM, 1928: 92).

Alfarería funeraria de tamaño reducido menciona Ampuero (1969) en tumbas adscritas a ì cultura inca-diaguita.

Aros cuadrangulares se hallaron en Angol, tanto en urnas como en sepulturas en posición tendida. Al sur del río Toltén, en Gorbea, excavamos varios de estos adornos en sus variantes con y sin muesca debajo del arco de suspensión (GoRdon y otros, 1972: 506). En la artualidad se los observa todavía, los hemos visto en Loncoche.

En la descripción de las joyas que servían de adorno de las mujeres indígenas en el siglo xvilı, el abate Molina dice:

"Llevan collares y manillas de cuentas de vidrio y zarcillas de plata de forma cuadrada" (Molina, I795: 163).

Aros circulares, pero de tamaño más reducido se hallaron en una urna en Angol, como también en el referido cementerio inca-diaguita.

En la bibliografía arqueológica no hemos encontrado referencia a tan pequeñas cuentas de piedra como las recolectadas en el fondo de la "canoa". Según una referencia de Medina (1882):

"las llancas eran más apreciadas mientras más pequeñas".

Tenemos una información sobre cl valor que los indígenas adscribian a los collares de piedras:

"Y las muertes que allí se han hecho las componen entre sí pagando a las partes... Y estas muertes se pagan siempre con llancas, que son las piedras verdes y negras, va. riadas con vetas de uno u otro color, que estiman más que los diamantes y esincraldas, de que no haccn caso. Y cada muerte se compone de diez pagas". (Ros.less, 1877: 133).

Fuera de los dientes de un niño de aproxi. madamente cinco años de edad, no se hallaron otros restos humanos dentro de la urna. Según los datos recogidos por Bullock, la gran mayoría de las urnas contenía restos de niños y solamente unas muy pocas contenian osamentas de mujeres de edad avanzada.

Aceptando el cantarito "pato" como símbolo femenino, podemos suponer que la urna contenía el cadáver de una niñita. Tal supo- 
sición se apoya en la presencia deI arco de suspensión de un aro, de un adorno femenino, aunque no exclusivo del sexo.

El cráneo y las osamentas en la "canoa" se identificaron como pertenecientes a un individuo adulto joven cuyo sexo no se determinó. Sin cmbargo, sus aclornos personitles, low seis aros y el collar de llancas perniten de. ducir, que lis sepultura guardaba los restos de una ınıjer.

La ubicación no anatómica de los huesos de las extremidades dentro de la sepultura indican que éstos han sido removidos, al $5 \mathrm{cl}$ depositadia la urna en medio de la "canoa". En cambio, sorprende cl hecho, que al mismo tiempo se la teniclo cuidado de no remover de su lugar al jarro fracturado ritualmente.

La gran cantidad de fragmentos alfareros y artetartos líticos indica, que las inhumaciones se efectuaron en terreno ocupacional. cerca de la vivienda, cn c] cementerio familiar y 10 comunal.

Martín Alonqueo nos informó también que los cadíreres de los niños de corta edad sc entcraban ditlás de la ruca, al cuidado de los perros prua critar gue sean robados en la noche para que se haga anchimallen de ellos. Tal tradición funcraria explicaúia la ausencia de urnas en comenterios comunales y la baja frecuencia de infantes on la población del cementerio de Gorbea. (Valdés. 1972: $590)$.

Otra tradición de disponer de los restos de infantes evidencian algunas scpulturas dobles. Rcymon (1971), halló una pequeña "canoa" dentro de unal cista. En Gorbea ubicanos una pequeña "canca" dentro de una más grancle. Un hallazgo descrito por Bullo:k (1970: 48) aparentemente corresponde a uná urna colocada en medio cle una sepultura en posición tendida (posiblemente "canoa"). En estos ejemplos se observa la inhumación de un niño dentro de la sepultura de un adulto. Podría suponerse que se trata de madre e hijo. Asi es que, en nuestro caso, en Padre Las Casas, la sepultura doble contendría el cadáver de una madre $\mathrm{y}$ de su hijo, quien falleció unos años después. Para sepultar al niño sc abrici la scpultura de la madre, se alcjaron 4.1, cxtremidades inferiores y la urna se coloci on posición anatomica, para dewher la riatura al seno de la madre.

De los ritos luneratios practicados registramos la colocación el: ofrenala junto al difunto. la olla seguramcute contenia alguna especie de alimento y el cántaro bebida fermentada. Se observó la práctica de fractura intencional de un jarro sobre una piedra plana (Foto 1). Guevara negó la existencia le cste rito mágico-religioso entre los aborigenes de Chile. Sin embargo, Latcham (1928: $39)$ y Cornely (1956:63). lo reconocicron en Itacana y en sepulturas diaguitas, respectivamente. Nosotros lo hemos observado en la mayoria de las sepulturas excaradas en lia región. Lamentablemente, ninguno de nuestros informantes consultados recordó el rito o su significado.

Las inhumaciones en la sepultura doble se efccuaron de acuerdo a dos tradiciones funerarias: en urua y ell "canoa". Al aborclar el posible origen de la "canoa" funeraria nos basamos en las condiciones ecolúgicas que cxistían en Chile central y on la región centromblir en épocas prehistólicas. lín esta. región boscosa los grandes áboles caílos, semiputrclactos. fácilmente podian ser acondicionados para las cabida y protección al cadaver. El empleo de troncos para tales clectos debc hahersc iniciado, en esta árca, mucho antes de la llegada de los conquistadores europeos.

La información de los cronistas Bibar y Conzález de Najera referente al rito fune1.ario inclígena, practicaclo en la región entre Sintiago y Concepción, eri la temprana épora de los primeros contactos de los españoles con la población autóctona, es de extraordinario valor para la investigación arqueológica. Ambos historiadores se refieren al empleo de "artesas" y "artesones" con fines funerarios. Los señalados artefactos de los cronistas 
se refieren al ataúd mapuche, al trolof labrado de un tronco en forma de canoa.

La preparación del trolof de un árbol vivo, era un trabajo difícil y demoroso. La selección de un árbol adecuado de considerable diámetro, de roble o de coigüe, el vol. teo y vaciado del tronco mediante artefactos líticos precisó la cooperación de un grupo dt personas, posiblemente voluntarios. En algunos casos el trabajo debía efectuarse lejos ic la habitación y demoraba varias semanas. No menos esfuerzo comunitario exigia el traslado del tronco ahuecado a la nuca del clifunto.

La referencia de los cronistas que tal artefacto se empleaba para la sepultación de "algunos principales" y de "caciques" permite considerar a la "canoa" funeraria como una distinción de jerarquía. Lamentablemente las crónicas no mencionan el rito de sepultación de la gente común.

No existe ninguna referencia al cmpleo de urnas de greda cocida por la población indigena para la inhumarión de sus muertos. El actual estado de la investigación arqueológica no permite vaticinar referente a la época o la cultura que introduce estos artefactos en la zona centro-sur del país. La afirmación de Guevara que su cmpleo se: anterior al de las "canoas" no se apoya en datos históricos o arqueológicos. La teoría de Bullock, de que pertenecerían a una cultura premapuche ha sido impugnada por Menghin.

Aunque la factura de una urna de greda exige un alto grado de dominio de la técnica alfarera, no reclama la colaboración de un grupo mayor de personas. Además, la vasija puede ser confeccionada en relativamente corto tiempo, en la misma ruca, por una o dos personas y no requiere la completa dedicación de los fabricantes.

Según Bullock, la gran mayoría de los res. tos humanos hallados en urnas pertenecían a infantes y solamente muy pocos correspondian a mujeres de edad avanzada. Es importante el hecho, que en ninguna urna se identificaron resto de varones.
Faron realizó estudios sociológicos en la moderna sociedad mapuche y obserró que las ceremonias funcrarias:

"varian de acuerdo a la posición social del difunto y de su familia. La edad, el sexo, la riqueza y el rango social son los principales determinantes de las celebraciones funerarias. Personas de elad madma reciben más o menos el mismo trato ceremonial... pero a los jefes principales y a los machis se dispensa mayor vistosidad. La sepultación de niños es generalmente más simple que la de las personas adultas; su pérdida para el grupo familiar no se considera tan grave como la de un adulto casado". (FARON, 1963: 143).

La sepultura tipo "canoa", o urna de la época prehistórica, es el reflejo de esta observación en la moderna comunidad indigena. A la llegada de los conquistadores europeos solamente algunos dignatarios indigenas se sepultaron en "canoa". Posteriormente, a fines del siglo $x i x$. según suponemos, con la mayor posibilidad de adquirir herramientas de metal y princijalmente con la radicación de los mapuches en reducciones, no solamente se facilita la manufactura del ataúd, sino su empleo se generaliza, se populariza. Sin embargo, la sepultación en "canoa" sigue reflejando cierta jerarquía, mayor riqueza del difunto o de su familia.

Los niños y las ancianas viudas, considerados de menor importancia dentro de la estructura socioeconómica indígena, no reciben la misma sepultación ceremonial que los miembros económicamente activos. Se los sepulta sin mayor ceremonia en urnas o grandes ollas detrás de la habitación. Todavía persiste la práctica y el recuerdo a la inhumación cle infantes detrás de la ruca, y Faron lo observó tal como nos la relató Martín Alonqueo. Aunque, aparentemente, no existe recuerdo del empleo de urnas entre los actuales mapuches, la señora Francisca Rupa. llán Huaiquiñix, de 36 años de edad, proce. 


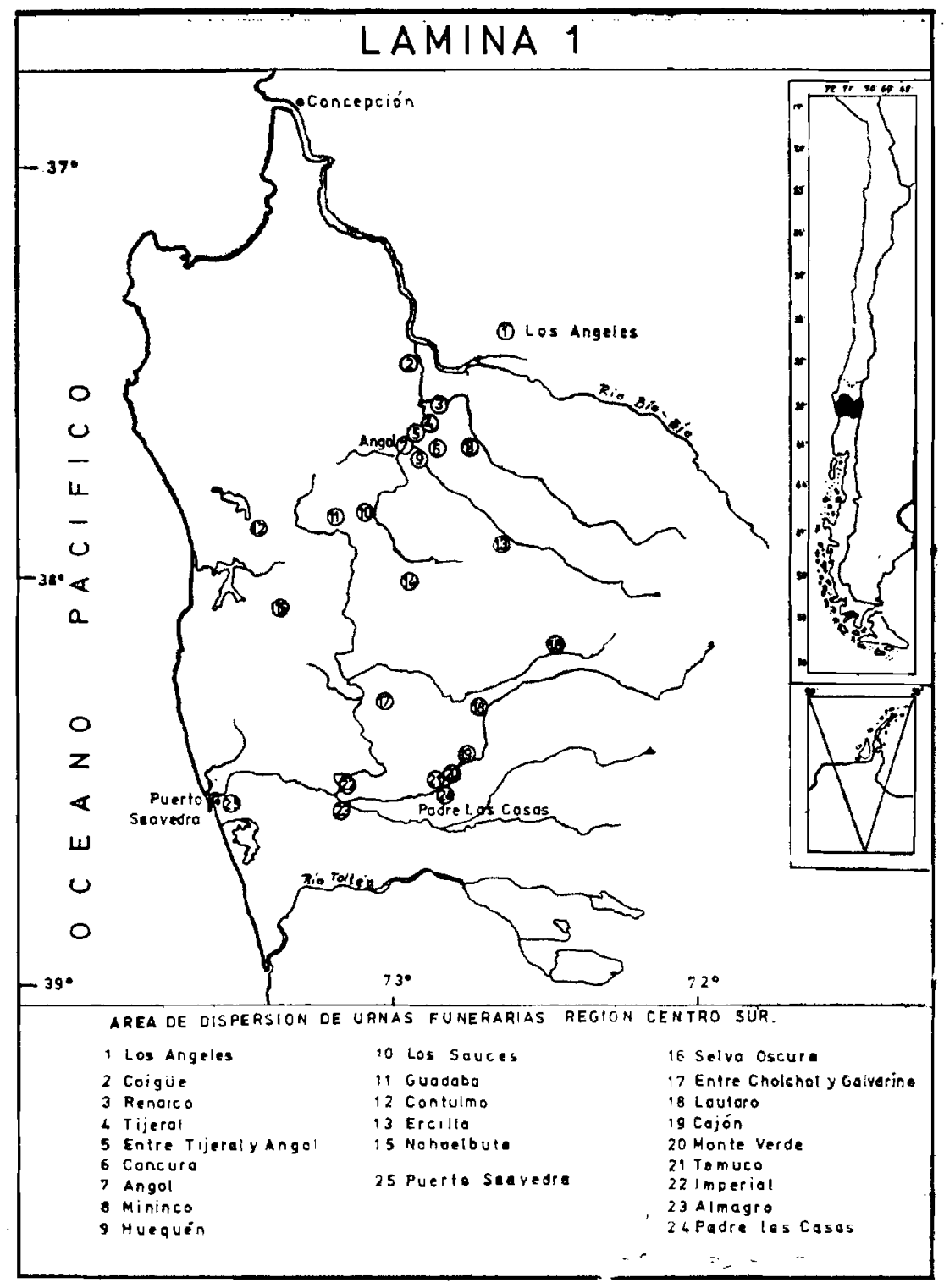

dente de la reducción Mallohue, ubicada cerca de la localidad de Barros Arana, al ve1 por primera vez la urna excavada no se atrevió a acercarse a ella. Al ser consultada por el motivo de su miedo, expresó: "En estas ollas grandes se sepultaba a los mapuches pobres". Nunca quiso revelar más al respecto.

Urnas de forma idéntica o de tipos similares a la excavada por nosotros se hallaron con relativa frecuencia en la ciudad de $\mathrm{Te}$ muco y sus cercanías. En el barrio Santa Rosa se halló uno de los pocos ejemplares de superficie decorada (Pino, 1969; Gordon,
1971 Ms.). Recientemente se descubrió un conjunto de cinco urnas en la Avenida Prie. to, lamentablemente destruidas por las pesadas palas mecánicas que emparejaban el terreno.

El área de dispersión de las urnas funerarias (Làm. 1), se extiende desde la orilla sur del río Cautín hasta los márgenes del río Biobío. Sin embargo, existen evidencias de vasijas funerarias con restos de infantes en las localidades de Curicó y Graneros, en el Valle Central; como también de esqueletos humanos conservados en urnas en Llolleo, en 


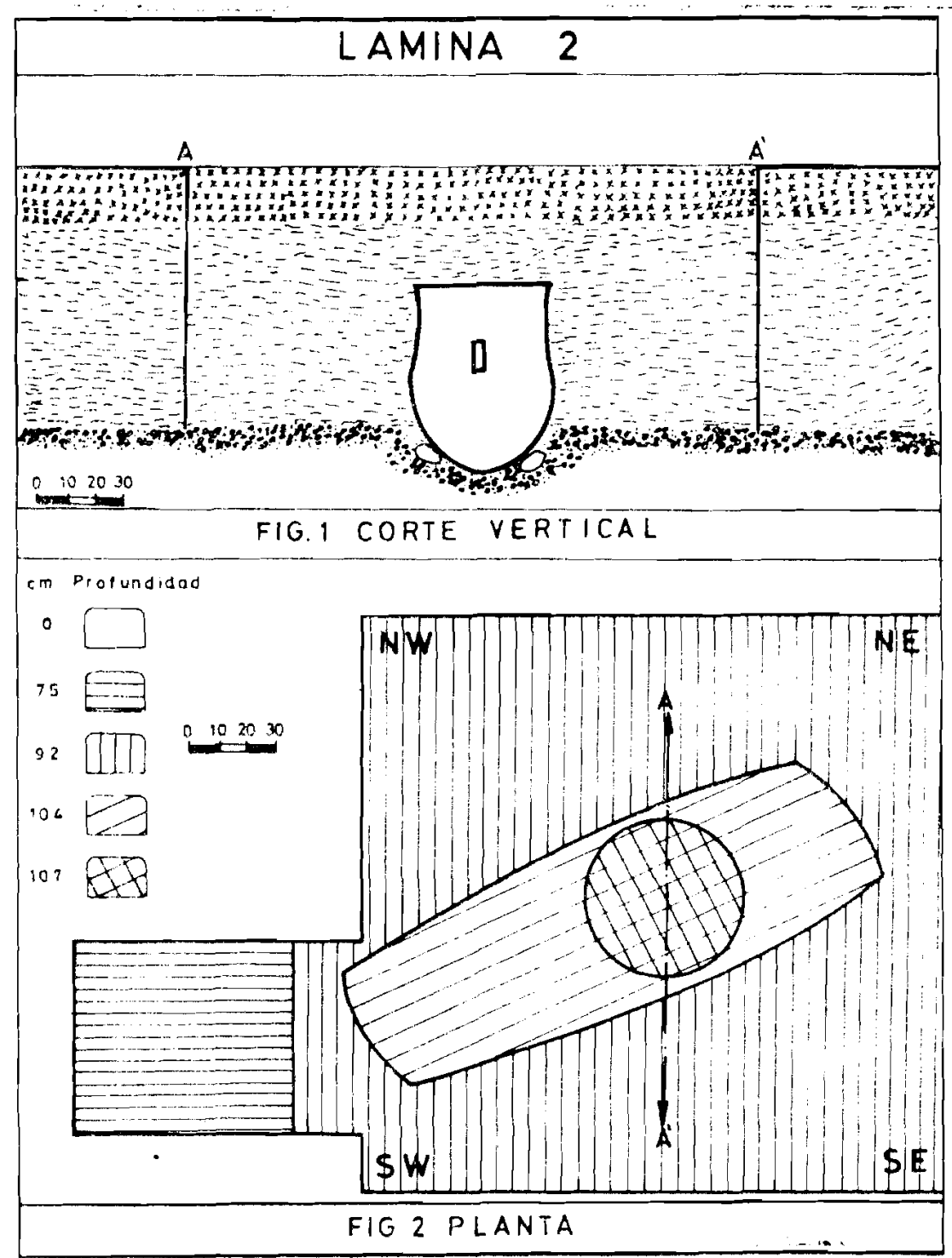

la costa central, y su presencia se proyecta hacia el Norte Chico.

\section{CAPITLEO VIJ}

\section{CRONOLOGIA}

Basándonos principalmente en ias condiciones meteorologicas de la región, que registra más de $1.300 \mathrm{~m} / \mathrm{m}$ de precipitaciones al año $y$ en informacioncs que coinciclian en sostener que el material óseo no se conserva en el suelo por más de 40 a 50 años. además de acuerdo con anteccdentes arqueologicos, hemos estimado en aproximarlimente 200 años la antiguiedad de ha sepultura doble. Contribuyó a tal suposición la presencia del cránco y de los huesos lareses, ammuce en mal estado de conservacion. como también el aro cuadrangular. frecuente en ef cenenterio de Grorbea y en uso torlaria. La alfarería presenta formas no registraclas en lat región Llamó la atención la forma arcaica cle la olla y la protuberancia horizontal sobre las asas. particularidad ausente en los yacimientos de 
Pitrén (Menghix, op. cit.), Challupen y Likan Ray (Cirvo, 1964), Gorbea (Gordon y otros, op. (it.), pero referido cu Alacanta : Lanco (Latcham, 1928) y Lanco (Gokdox, IIs. 1971). Supusimos que la falta de alfarería de tipo "Valclivia" en las sepulturas se debió al azar.

Gracias al gentil ofrecimiento del Director de! Laboratorio de Racliocarbono Gif-surYrette, Francia, G. Delibrias, ha sido posible fechar, mediante el método de radiocarbono, una muestra de carbón regetal recolectada en la sepultura en "canoa", cuya colad se determinó:

Gif-3841 $670 \pm 80$ años A.P. $1280 \pm 80$ D.C

Esta edad es muy anterior a lo estimado y deberta ser confirmada mediante nuevas determinaciones, que podrían efectuarse sobre los huesos remiticlos al mismo laboratorio o mediante la termoluminiscencia de fragmentos de la urna.

Futuras determinaciones cronológicas confirmarán o rechazarán la validez de esta primera fecha radiocarbónica de una fase cultural prehistórica del área centro-sur del pais.

\section{CAPITULO IX}

\section{CONCLUSIONES}

a) La excaración de salvamento permitió reconocer un sitio ocupacional y funerario;

b) La distribución estratigráfica cuantitativa del material cultural indica una acumulación gradual prolongada de objetos de factura inclígena, interrumpida súbitamente y reemplazado por elementos de nuestra cultura del siglo $\mathbf{x x}$;

c) El contexto de la sepultura doble, la asociación directa de "canoa" y urna funeraria atestigua el einpleo simultáneo de los dos atrefactos funerarios por la misma comunidad. Su empleo respectivo está condicionado por la posición social del difunto. Las urnas son empleaclas para la inhumación de infantes y ancianas, mientras las "canoas" para indivicluos de alguna inportancia dentro de lit comunidad indigena;

d) De acuerclo con la eclacl absoluta de la muestra Gif $384 \mathrm{I}$ de $670 \pm 80$ años antes del presente, las scpulturas pueden ser adscritas a una cultura agroalfarera en una fase tardia prelistórica, y

e) El csiado artual de la investigación ar. queológicil del área centro-sur de Chile no p-rmice ninguma conclusión delinitiva respecto de la época y cultura que introduce las urnas funcrurias en la región.

\section{ANEXO 1}

Informe sobre restos óseos humanos del sitio Padre Las Casas, provincia de Cautín. IX Región, Chile.

Consuelo Valdés $\mathrm{Ch}$.

Trabajo realizado en el Laboratorio de Antropología Física del Departamento de Ciencias Antropológicas y Arqueológicas, Facultad de Ciencias Humanas, Universidad de Chile, Sede Oriente, bajo la dirección del profesor Juan R. Munizaga, durante el segundo semestre de 1975 .

Item No 14. Diáfisis de fémur, probablemente derecho de un individuo adulto; en mal estado de conservación.

Item No 26. Fragmentos de huesos largos. sin identificar.

ltem $N^{\circ}$ 27. Diáfisis fémur izquierdo, individuo adulto; mal estado de conservación.

Item $N^{\circ}$ 28. Diafisis tibia derecha, individuo adulto; mal estado de conservación.

Item $35 \mathrm{~A}$. Fragmentos de bóveda craneana. Están presentẹ fragmentos 
de los huesos parietal, frontal y temporales. Por el tamaño y estado de las suturas correspondería a un adulto joven.

Item 35B. Dentadura mixta: 12 molares, 8 premolares, 4 caninos y 8 incisivos.

Primer molar con desgaste mínimo.

Incisivos en forma de pala. 20 definitivos y 12 temporales. En relación a patología, no se observan caries, pero un incisivo lateral inferior presenta una cresta media en la superficie vestibular susceptible de ser considerada patológica.

Item $N^{0} 1 . \quad$ Dientes. Se encuentran presen. tes 6 incisivos, 3 primer molar temporal, 3 segundo molar temporal: Estos dientes no presentan desgaste. Edad aproximada 3 a 5 años.

\section{COMENTARIOS:}

Los restos humanos corresponden por lo menos a dos individuos. Uno de ellos representado por dientes, fragmentos de huesos largos y de bóveda craneana que pertenecerían a un adulto joven, con dentadura de tipo mongoloicle. El otro, a un niño de 3 a 5 años de edad, representado por algunos dientes que indican su afinidad mongoloide. En ambos casos no se pudo identificar el sexo.

\section{ANEXO 2}

Informe sobre piezas dentarias procedentes de Padre Las Casas.

\section{Dr. Patricio Urquieta}

\section{A. Individuo adulto}

Piezas dentarias en muy mal estado de conservación, se mantienen solamente las coronas de Jos dientes debido, tal vez, a la humedad del terreno. Presentes 31 piezas den. tarias, ausente solamente un incisivo central inferior.

Lno de los terceros molares superiores presenta una coloración vercle, por algún con. tacto externo post mortem.

Los segundos molares inferiores presentan un típico patrón oclusal +4 .

Un incisivo lateral inferior tiene en su cara vestibular un rodete adamantino, segu. ramente conclicionaclo genéticamente.

En general, las piezas dentarias no se presentan abrasionadas, salvo el caso de incisivos y primeros molares con una abrasión de tipo plano marcada.

De los 31 dientes, existen dos caries en los terceros molares superiores.

\section{B. Niño}

Restos en muy mal estado de conservación, se podría pensar en una edad aproximada de 5 años, basánclose en el nivel de calcificación de los primeros molares y caninos inferiores.

Se conservan las coronas de incisivos laterales superiores, segundos molares superiores temporales y primeros molares. Un incisivo central inferior, ambos incisivos laterales, caninos inferiores, molares temporales inferiores derecho y el primer molar inferior del mismo lado.

Aparentemente las piezas dentarias perma. nentes no habrían erupcionado.

BIBLIOGR AFIA

Ampuero B., Gonzalo. Excayaciones arqueológicas en el fundo "Coquimbo", Departamento de La Serena. Actas $v$ Congreso Nacional de Arqueología.
Dirección General de Bibliotecas, Museos y Archivos. Museo Arqueológico de La Serena. La Serena, 1969, pp. 153-166. 
Augusta, Fray Félix DF. Diccionario Araucano. Imprenta y Editorial "San Francisco". Padre Las Casits. Chile, 1960

Bfrdichewsisy S., Bernardo. I.xcavaciones en lia "Cue" va re los Catalanes". Boletín de Prehistoria de Clisle. Año 1. X' 1. Departamento de Historia. Facultad de Filosofía y Educación. Universidad de Chile. Santiago, 1968, pp. 33.83

Berdichewsky S., Brerizdo y Calyo de G., Mayo. Excavaciones en comenterios indigenas de la región de Calalquén. Actas del vi Congreso de Arqueolo. gía Chilena. Lniversidad de Chile. Departamento du Cicncias Antropológicas y Avqueología. Socicdad Chilena de Arqueología. Sartiago, 1972, pp. 529-558.

Bibar, Gerónimo de. Crónica y relación copiosa y ver dadera de los Reynos de Chile. (Mdoviu). Fondo Flistónico y Bibliográfico José Toribio Medina. Santiago, 1966.

Bellock, Dilmax $S$. Utnas funerarias prehistóricás de la región de Angol. Bolctín del Museo Nacional de Ilistoria Natural, xxv1. Santiago, 1955, pp. 73-15\%.

Bullock, Dil.alax S. Linas funcrarias prehistóricas de la region de Angol. Botetín de la Sociedad de Bio. logía de Cuncepción. Tomo xulli. Concepción. Chi le, 1970

Calro de G., Mayo. Exploración arqueológica de la región norte del lago Calafquén. Comuna de Panguipulli. Prov de Valdivia. Arqueología de Chile Central y Areas Vecinas. Santiago, 1964, pp. 178 181 .

Casaniqufia, Robolfo. Nota sobre sitios y piedras rituales dè ámbito pehuenche austral. Actas del vi Congreso de Arqueología de Chile. Departa mento de Ciencias Antropológicas y Arqueología Sociedad Chilena de Arqueología. Santiago, 1972 pp, $487-500$.

Chizelle B., Gabrielle; Coronado, Luis y Seguel, ZuLEMA. Excavación de saivamento en la localidad de Chiguayante. Prov. Concepción. Actas del $v$ Con. greso Nacional de Arqueología. Museo Arqueológi co de La Serena. La Serena, 1969, pp. 351-375

Confo. Suelos. Descripciones (Proyecto aereofotogramétrico, Chile) . oEA/Bid. Instituto de Investigación de Recursos Naturales. Corfo. Publicación No 2. 1964.
Cornety, lrancisco. Cultura diaguita chilena y culturat de FI Molle. Santiago, 1956.

1) Ilfehty, Tom 1). Small archacological site invesigations for interpretation of site activities. Reprint. Bulletin of the Texas Archaeological Society. Vol. 44. 1973 .

Finos, L.ts C. Mapuche Social Structure, Illinois Studics in Anthropology No 1. The University of Illinois l'ress. I'rbana. 1961.

Farox, I.tis C. Deatl and Fertility Rites of the Mapuche (Araucanian) Indians of Contral Chile. Fthnolog! Vol. 11. No 2. 1963, pp. 135-156

Farox, Lew $C$. The Mapuche Indians of Chile. Case Studies in Cultural Antlıropology. New Y'ork. 1968.

Gonzílez di líjera, Aloxso. Desengaño y reparo de la gucrra dol Reino de Chile. Editorial Andrés Bello. Santiago, 1971 .

Gordox, AMerico. Descripción de una urna funeraria decorada hallada en-Temuco. Ms, 1971.

GORDOx, Axírico. Informe sobre la excavación de una sepultura en l.oncocle, Departamento de Villarri ca, provincial de Cautín. Ms. 1973.

Gordon, Asirico; Madrid, Jacelelin y Monleón de, Juma. 1972. Excavación del cementerio indigena en Gorbea (Sitio Go-3), provincia de Cautín, Chile. Informe preliminar. Actas del vi Congreso de $\mathrm{Ar}$ queología Chilena. Liniversidad de Chile. Depto. de Ciencias Antropológicas y Arqueología. Sociedad Cliitena de Arqueología. Santiago, 1972, pp. 501 514.

Glevara, Tomás. Psicologia del pueblo araucano. San. tiago, 1908.

Gervart, Touśs. La mentalidad araucana. Tomo vill, Santiago, Valparaíso, 1910.

Gusinde, MarTíx, Stcinkistengräber im Gebiet der sïdlichen Araukaner, Sep. Actes de xxym Congres International des Americanistes. Paris, 1947. Mu sée de l'Homme. Paris. 1948, pp. 609-614.

Latcham, Ricardo E. Costumbres mortuorias de los indios de Chile y otras partes de América. Anales de la Universidad de Chile. Santiago, 1915.

Latcham, Ricardo E. La organización social y las 
crecncias religiosas de los antiguos arancanos. Publicación Museo Etnológico : Antropológico. San tiago, 1924.

Latchas, Ricardo E. I a alfareria indigena chiena. Santiago, 1928.

Latchan, Ricardo E. Prehistoria indigena chilena. Oficina del Libro. Santiago, 1936.

Medna, José Toribio. Los aborigenes de Chile (1882). Fondo Histórico y Bibliográfico José Toribio Medina. Santiago, 1952.

Mencix, Osvaldo A. F. Estudios de Prehistoria arau. cana. Sep. Studia Praehistorica 11. Centro Argentino de Estudios Prelistóricos. Buenos Aires, 196?

Molina, Juan Ignacio. Compendio de la Historia Ci. vil del Reyno de Chile. Parte 1I. Madrid, 1795

Mostny, Grete. Ideas Mágico-Religiosas de los "Atacamas". Boletín del Museo Nacional de Historia Natural. Tomo xxx. Santiago, 196 7 , pp. 199.145

Mostry, Grete. Prehistoria de Chile. Editorial Universitaria, S. A. Santiago, 197 I.
Orakzix, Atrelduio. Los hjoekkemocidinger de Jas costas de Melipilla y Casablanca 1910.

Pixo Z., Edcardo. Contribución al estudio de la cul tura premapuchc. Rehuc $N$ ? 2. Universidad de Concepcón. Conceprión. 1969, p?. 85.90.

Reymond, Jacqueline. Cementerio araucano de Mem. brillo. Boletín de Prehistoria de Chile. Año 3. No 4. Depto. de Ciencias Antropológicas y Arqueológicas. Lniversidad de Clilt: Santiago, 1971.

Rosales, Dieco de. Historia General de el Reino de Chile. Flandes Indiano (1674). Editorial Lniversi. taria, S. A. Santiago, 1969 .

Valdés Ch., Conscelo. Restos óseos humanos de un comenterio indigena, Gorbea, provincia de Cautín. Chile. Actas vi Congreso de Arqueologia Cliblena, Universidad de Chile, Depto. de Ciencias Antropológicas y Arqueologia. Santiago, 1972, pp. 5lú-522.

Van der Maele, Malricio. Excayaciones de cemente. rios, fogones y tačitas de la regiún de Valdivia. Investigaciones Históricas. Investigaciones Arqueológicas. Universidad Austral de Chile. Valdivia, Chile, 1968. 